\title{
Üher Fermentlähmung.
}

II. Mitteilung.

Von

L. Lichtwitz.

(Aus der medizin. Klinik und dem landwirtschaftlich-bakteriolog. Institut zu Göttingen.)

(Der Redaktion zugegangen am 8. April 1915.)

Mit einer Kurvenzeichnung.

Im 78. Bande dieser Zeitschrift') habe ich über Versuche berichtet, aus denen hervorging, daß Hefe, die in einer Lösung von Rohrzucker + Invertzucker gewachsen war, eine geringere Inversionskraft hatte, als nur mit Rohrzuckerlösung gezogene Hefe, und daß diese Abnahme an Inversionsvermögen, die als Fermentlähmung bezeichnet wurde, auch nach Fortschaffung des Invertzuckers (durch Auswaschen oder Vergären) bestehen blieb.

Diesen Versuchen ist widersprochen worden, zuerst von Meisenheimer, Gambarjan und Semper, ${ }^{2}$ ) die aus Versuchen ganz anderer Art auch ganz andere Resultate bekommen haben. Ich habe auf das Unzutreffende der Schlußfolgerungen dieser Autoren hingewiesen, ${ }^{8}$ ) und darf wohl das Schweigen zu meiner Kritik als Zustimmung auffassen. Auch Euler und Cramer') haben die Frage der Fermentbeeinflussung durch Rohrzucker einerseits, durch Glukose anderseits studiert und nichts von Fermentlähmung gefunden. Die

1) Diese Zeitschrift, Bd. 78, S. 128, 1912.

2) Biochem. Zeitschrift, Bd. 54, S. 112, 1913.

3) Biochem. Zeitschrift, Bd. 56, S. 160, 1913.

4) Diese Zeitschrift, Bd. 88, S. 430, 1913; Bd. 89, S. 277, 1914. 
Versuche von Euler und Cramer unterscheiden sich in zwei Punkten von den meinigen. Erstens ist die Zahl der verimpften Hefezellen in ihren Versuchen sehr groß $(1 \mathrm{~g}$ frische Hefe $=18 \times 10^{10}$ Zellen für $100 \mathrm{ccm}$ Nährlösung gegenüber 8 Millionen Zellen in meinen früheren und in den nachstehenden Versuchen). Zweitens aber haben Euler und Cramer nur mit Lösungen eines Zuckers gearbeitet, während ich die Fermentlähmung nur bei Hefe aus Lösungen von Rohrzucker + Invertzucker, bezw. Rohrzucker + Glukose (oder Lävulose) gefunden habe. ${ }^{1}$ )

Da dieser Unterschied für das Ergebnis des Versuches wesentlich ist, und da sich daraus die Möglichkeit einer Einsicht in die physikalisch-chemischen Bedingungen ergibt, die der Fermentlähmung zugrunde liegen, so soll eine neue Versuchsreihe mitgeteilt werden.

Gemäß dem Typus II der früheren Versuche wurden 14 Gärversuche von je $100 \mathrm{ccm}$ Hefewasser angesetzt. Jedes Glas enthielt Zucker und zwar nur Rohrzucker oder nur Invertzucker, oder Mischungen beider in verschiedenen Verhältnissen. Die Zuckermenge, als Invertzucker berechnet, betrug in jedem Glase $20 \mathrm{~g}$. Der Kopf der nachstehenden Tabelle macht die Verteilung der Zucker deutlich. Alle Kulturen wurden mit je 8 Millionen Hefezelle (Reinkultur der Weinhefe «OppenheimerKreuz s) geimpft und zunächst der Gärungsverlauf gemessen.

Diese Versuchsreihe hat, wie Tabelle und Kurve zeigen, das nämliche Ergebnis, wie die früher mitgeteilten Versuche. Die Vergärung der reinen Rohrzuckerlösung und der reinen Invertzuckerlösung verläuft geradlinig, während die Mischzuckerlösungen einen scharfen Knick in der Kurve der $\mathrm{CO}_{2}$ Entwicklung abgeben, der ganz genau dort liegt, wo die aus dem Invertzucker berechnete Kohlensäure entwichen ist. Von da an verläuft die Gärung mit gleichbleibender Trägheit, und um so langsamer, je höher die Konzentration des primär zugesetzten Invertzuckers war.

Daß in solchen Kulturversuchen Inversionsgeschwin-

1) Herr von Euler und ich haben nach brieflicher Verständigung diese Frage weiter verfolgt. Nach einer mündlichen Mitteilung von Herrn von Euler stimmen die tatsächlichen Ergebnisse unserer Versuche nunmehr überein. 
Tabelle 1.

\begin{tabular}{|c|c|c|c|c|c|c|c|c|c|c|c|c|c|c|c|}
\hline & & I. & II. & III. & IV. & V. & VI. & VII. V & VIII. & IX. & $\mathrm{x}$. & XI. & XII. & XIII. & XIV. \\
\hline Rohrz & ucker & 19,0 & 19,0 & 11,4 & 11,4 & 9,5 & 9,5 & 7,6 & 7,6 & 9,5 & 9,5 & 9,5 & 9,5 & 0 & 0 \\
\hline Dext & rose & 0 & 0 & 4,0 & 4,0 & 5,0 & 5,0 & 6,0 & 6,0 & 10,0 & 10,0 & 0 & 0 & 10,0 & 10,0 \\
\hline Läru & lose & 0 & 0 & 4,0 & 4,0 & 5,0 & 5,0 & 6,0 & 6,0 & 0 & 0 & 10,0 & $|10,0|$ & 10,0 & 10,0 \\
\hline $\begin{array}{r}\mathrm{Ze} \\
\mathrm{Tag}\end{array}$ & and. & & & & & & & C & $\mathrm{CO}_{2}$ & & & & & & \\
\hline I. 1. & 615 & & np & & & & & & & & & & & & \\
\hline I. & $62^{30}$ & $|1,53|$ & 1,5 & 1,35 & 1,33 & $|1,07|$ & 1,22 & $|1,40| 1$ & 1,57 & $|1,65|$ & 1,90 & 0,87 & 0,70 & 0,77 & 1,32 \\
\hline 16. I. & 8640 & 2,63 & $2,62 \mid$ & $|2,30|_{2}$ & 2,20 & $|1,97|_{2}$ & 2,17 & $2,40 \mid 2$ & 2,72 & $2,67 \mid$ & 2,75 & 1,72 & 1,60 & 1,70 & 2,47 \\
\hline 17. I. & $108^{30}$ & 3,65 & $3,63 \mid$ & $2,97 \mid$ & 2,90 & $|2,70|$ & 2,89 & 253 & 3,49 & $3,28 \mid$ & 3,50 & 2,47 & 2,42 & 2,64 & 3,40 \\
\hline 18. I. & 13230 & $4,47 \mid$ & 4,58 & $|3,53|$ & 3,57 & $|3,49|$ & 3,69 & 4,02 & 4,22 & 3,87 & 4,00 & 3,37 & 3,05 & 3,50 & 4,25 \\
\hline 19. I. & 15630 & 5,25 & $5,60 \mid$ & 3,90 & 3,90 & $4,22 \mid$ & 4,32 & $4,57 \mid 4$ & 4,77 & 4,35 & 4,20 & 4,12 & 3,98 & 4,50 & 4,95 \\
\hline 20. I. & 18030 & 6,25 & 6,58 & 4 & 4,30 & 4,85 & 4,90 & 5 & 5,27 & 4,48 & 4,38 & 4,87 & 4,78 & 5,37 & 5,82 \\
\hline 21. I. & 20430 & 6,95 & 7,28 & 4,70 & 4,72 & $5,20 \mid 5$ & 5,39 & $\overline{0}, \overline{5} 7 \mid \tilde{5}$ & 5,67 & 4,70 & 4,60 & 5,42 & 5,60 & 6,27 & 6,60 \\
\hline 22. 1 . & 22830 & 7,32 & $|7,80|$ & $|4,85| 4$ & 4,85 & $|5,42|$ & 5,52 & $5,60 \mid 5$ & 5,67 & 4,75 & 4,60 & 5,59 & 6,00 & 6,82 & 7,10 \\
\hline 23. I. & 25230 & 7,73 & $8,25 \mid$ & 5,03 & 5,17 & $|5,65|$ & 5,79 & 5,75 & 5,82 & 4,80 & 4,65 & 5,77 & 6,38 & 7,29 & 7,60 \\
\hline 24. I. & $276^{30}$ & 8,13 & $8,65 \mid$ & 1 & 5,38 & $5,87]_{6}$ & 6,04 & 85 & 5,97 & 4,83 & 4,75 & 5,97 & 6,75 & 7,80 & 7,99 \\
\hline 26. 1. & 32430 & 8,83 & 9,15 & 5,68 & 5,98 & $6,39 \mid 6$ & 6,60 & $6,10 \mid 6$ & 6,30 & 5,10 & 5,03 & 6,49 & 7,38 & 8,44 & 8,49 \\
\hline 28. I. & 37230 & 9,03 & 9,30 & $6,25 \mid 6$ & 6,40 & 6,72 & 6,97 & $6,30.6$ & 6,45 & 5,25 & 5,15 & 6,75 & 7,55 & 8,52 & 8,52 \\
\hline 31. 1 . & $444^{30}$ & 9,17 & 9,50 & 6,90 & 7,00 & $7,27\}$ & 7,57 & 6,606 & 6,77 & 5,55 & 5,43 & 7,22 & 8,03 & 8,57 & 8,55 \\
\hline 3. 11 . & 517 & 9,27 & 9,68 & $|7,60|^{7}$ & $7,63^{*}$ & $|7,67| \varepsilon$ & $8,07 *$ & 6,95 & $7,03 *$ & 5,92 & 5,85 & 7,64 & 8,40 & 8,59 & 8,62 \\
\hline 7. II. & 613 & 9,27 & 9,68 & $8,15 \mid$ & - & 8,12 & - & 7,32 & - & 6,47 & 6,30 & 8,17 & 8,62 & 8,65 & 8,67 \\
\hline 11. II. & 709 & - & - & $8,60 \mid$ & - & 8,49 & - & 7,85 & - & 6,85 & 6,67 & 8,34 & 8,68 & 8,65 & 8,67 \\
\hline 14. II. & 805 & - & - & 8,88 & - & $8,70 \mid$ & - & 7,95 & - & 7,20 & 7,07 & 8,57 & 8,80 & - & - \\
\hline 19. II. & 925 & - & - & 9,02 & - & 8,90 & - & 8,20 & - & 7,82 & 7,42 & 8,72 & - & - & - \\
\hline 25. II. & 1069 & - & - & 9,30 & - & 9,02 & - & 8,55 & - & 8,25 & 7,82 & 8,92 & 8,85 & - & - \\
\hline 2. III. & $128 \overline{5}$ & - & - & 9,30 & - & 9,05 & - & 8,68 & - & 8,55 & 8,17 & 8,92 & - & - & - \\
\hline 9. III. & 1353 & - & - & - & - & - & - & 8,87 & - & 8,83 & 8,52 & - & 8,85 & - & - \\
\hline 20. III. & 1617 & $1-$ & - & - & - & - & - & - & - & $|9,00|$ & $|8,77|$ & - & - & - & - \\
\hline
\end{tabular}

- Versuch abgebrochen zum Zwecke der Zuckeranalyse. 
digkeit und Vergärungsgeschwindigkeit parallel gehen, daß in einem System von Fermenten und Substraten eine strenge Ordnung herrscht, ist früher gezeigt worden.

Auch in dieser Versuchsreihe wurde in einigen, auf der Tabelle mit * bezeichneten Proben die Zuckeranalyse gemacht und zwar zu einer Zeit, als die Vergärung der reinen Rohrzuckerlösung und der reinen Invertzuckerlösung beendet war.

\begin{tabular}{c|c|c|c}
\hline \hline \multirow{2}{*}{ Es enthielt Glas } & \multirow{2}{*}{ am } & \multicolumn{2}{|c}{ reduzierender Zucker } \\
& & vor der Hydrolyse & nach der Hydrolyse \\
\hline Nr. IV & 3. II. & $0,298 \mathrm{~g}$ & $3,460 \mathrm{~g}$ \\
× VI & 3. II. & 0,242, & 2,410, \\
٪ VIII & 3. Il. & 0,250, & 3,448,
\end{tabular}

Auch an diesen Stichproben, ausführlicher an Versuch III. der I. Mitteilung, ist die Langsamkeit der Inversion zu sehen, die die Langsamkeit der Gärung zur Folge hat.

Es wurden nun die in den reinen Rohrzuckerlösungen (Glas I und II) und in den reinen Invertzuckerlösungen gewachsenen Hefen von den ausgegorenen Flüssigkeiten getrennt und in neuen Gärversuchen mit je $100 \mathrm{ccm}$ eines $19 \mathrm{~g}$ Rohrzucker enthaltenden Hefewassers angesetzt.

Tabelle 2.

\begin{tabular}{l|c|c|c|c|c}
\hline \hline \multirow{2}{*}{ Tag } & \multirow{2}{*}{ Stunden } & \multicolumn{5}{|c}{ g CO } \\
& & Glas I & Glas II & Glas III & Glas IV \\
\hline 16. II. & - & - & - & - & - \\
19. II. & $63^{30}$ & 2,00 & 1,83 & 1,72 & 1,77 \\
20. II. & 8730 & 3,15 & 2,85 & 2,60 & 2,82 \\
21. II. & $111^{30}$ & 4,10 & 3,95 & 3,67 & 3,92 \\
23. II. & 15930 & 5,80 & 5,55 & 5,27 & 5,62 \\
24. II. & $183^{30}$ & 6,20 & 6,05 & 5,72 & 6,22 \\
25. II. & $207^{30}$ & 6,70 & 6,55 & 6,27 & 6,92 \\
26. II. & $231^{30}$ & 7,20 & 6,95 & 6,67 & 7,32 \\
27. II. & $255^{30}$ & 7,40 & 7,15 & 6,97 & 7,42 \\
28. II. & 27930 & 7,60 & 7,30 & 7,27 & 7,62 \\
2. III. & 32730 & 7,80 & 7,60 & 7,67 & 7,77 \\
4. III. & $375^{30}$ & 8,27 & 8,00 & 8,22 & 8,22
\end{tabular}



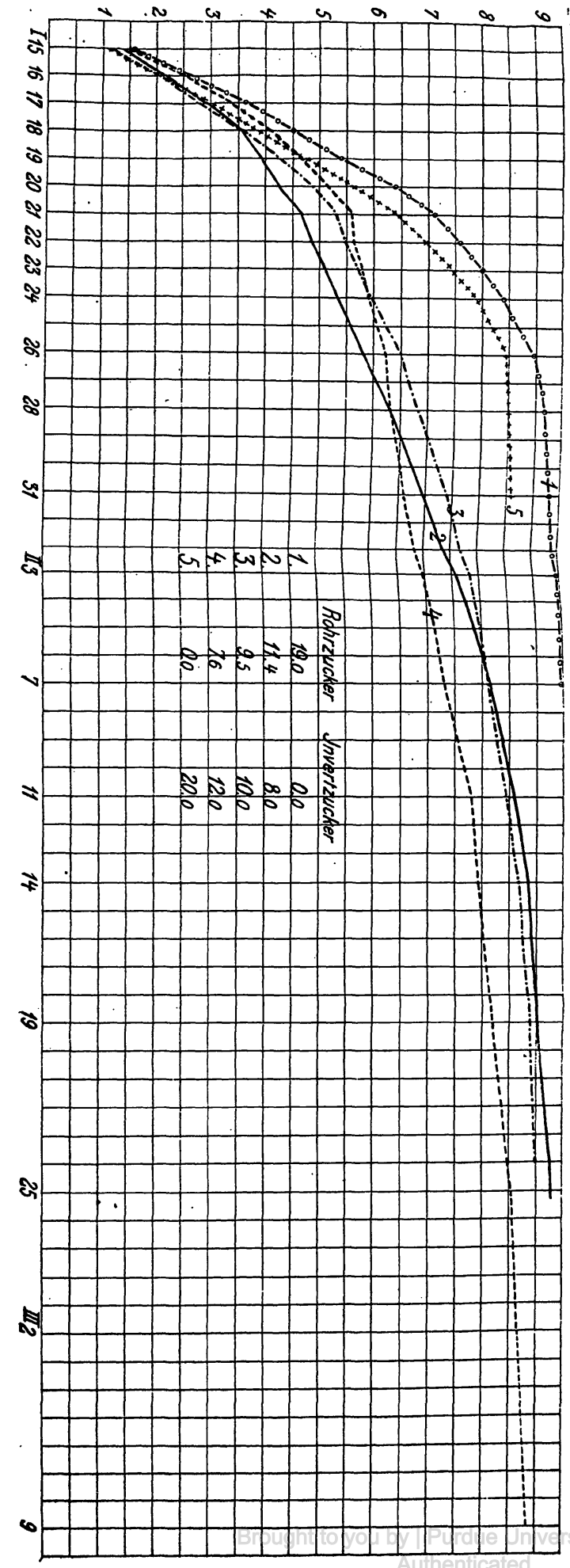
In allen 4 Proben erfolgte die Gärung gleich schnell. Da man, wie die früheren Versuche zeigen (in Kulturversuchen), aus der Gärungsgeschwindigkeit auf die Inversionsgeschwindigkeit schließen kann, so zeigt dieser Versuch in Übereinstimmung mit Euler und Cramer, daB das Wachstum der Hefe in reinen Rohrzucker- und reinen Invertzuckerlösungen (oder die Vorbehandlung der Hefe mit solchen Lösungen) auf die Inrertase ohne jeden Einfluß ist.

Es ist also nicht die Anwesenheit der durch die Fermenttätigkeit entstehenden Produkte schlechthin, die das Ferment schädigt, sondern nur ihre Anwesenheit bei Gegenwart des Substrates. So weit das Tatsächliche.

Die Vorstellung, die man sich von den physikalischchemischen Vorgängen macht, die der Fermentlähmung $z u$ Grunde liegen, muß bei der Unbestimmtheit der chemischen und physikalischen Natur eines Ferments eine Lücke haben. Es erscheint aber möglich wenigstens den Ort zu finden, an der vorläufig die Erkenntnis aufhört.

Wählen wir zar Verdeutlichung als Beispiel den vorliegenden Fall Rohrzucker ( $R z$ )-Invertase (I)-Invertzucker ( $D L=$ Dextrose, Lävulose), so ist ein logisches Erfordernis, $\mathrm{da} B$ Rohrzucker und Invertase in eine enge rüumliche $\mathrm{Be}$ ziehung treten, also einen Komplex RI bilden, in dem die Wirkung der Invertase vor sich geht. Durch diese Wirkung entsteht aus RI der Komplex Invertzucker-Invertase DLI, der sich dann in seine Einzelbestandteile auföst (dissoziiert). Als chemische Gleichung würde sich also ergeben:

$$
\mathrm{R}+\mathrm{I} \longrightarrow \mathrm{RI} \longrightarrow \mathrm{DLI} \longrightarrow \mathrm{D}+\mathrm{L}+\mathrm{I} \text {. }
$$

Dieser letzte Vorgang, die Dissoziation, muß aber nach bekannten Gesetzen gehemmt werden, wenn in der Lösung $D$ oder $L$ oder $D+L$ vorhanden ist. $D a B$ in dem Komplex $R I$ die Spaltung des Rohrzuckers bei Gegenwart von Invertzucker nicht oder nur langsam erfolgte, wäre physikalisch-chemisch nicht $z u$ begründen. 
Bis zu dem Prozeß der Dissoziation können wir dem Vorgang folgen. Warum aber, wenn bei Gegenwart von viel Invertzucker in der Lösung der Komplex DIJl längere Zeit besteht, das Ferment geschwächt ist, ob es dadurch chemisch verändert wird oder ob sich der Komplex DLI auf eine dauernde trägere Dissoziation einstellt, entzieht sich dem Verständnis.

Göttingen, 28. März 191 Ј.

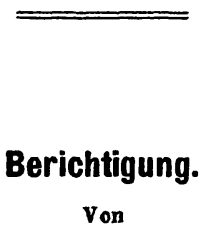

Dr. Max Oppenheimer.

(Der Redaktion zugegangen am 22. April 1915.)

Ich bemerke jetzt erst, daß sich in meine zweite Mitteilung \&Über die Bildung von Milchsäure bei der alkoholischen Gärung, ') ein bedauerlicher Fehler eingeschlichen hat, welcher weder im Manuskript noch im Korrekturabzug vorhanden war.

Es muß auf Seite 267 selbstverständlich heißen:

snnerers Cannizzaro:

und nicht:

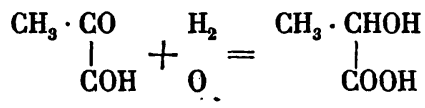

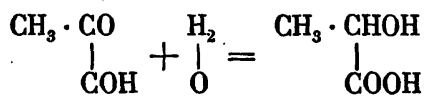

Durch die richtige Formulierung sollte ja gerade die gekoppelte Reaktion von der Neubergschen Pseudo-Wasseraufnahme unterschieden werden, wie übrigens aus dem Texte zur Genüge hervorgeht.

1) Max Oppenheimer, Diese Zeitschrift, Bd. 93, S. 262 (1914). 